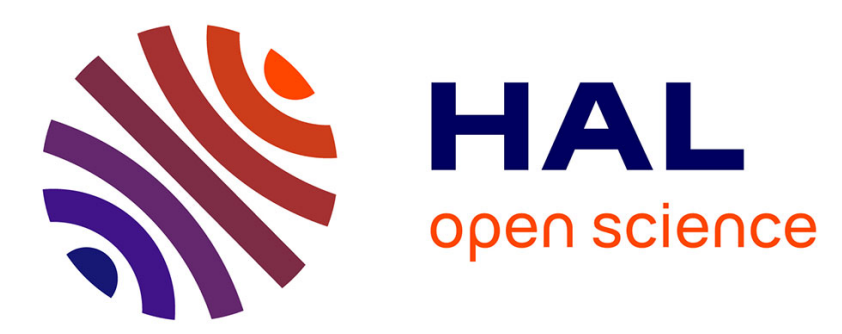

\title{
Approche méthodologique et application populationnelle des adaptations musculaires isocinétiques
}

Pierre Louis Bernard, Hubert Blain, Guillaume Tallon, Sofiane Ramdani

\section{To cite this version:}

Pierre Louis Bernard, Hubert Blain, Guillaume Tallon, Sofiane Ramdani. Approche méthodologique et application populationnelle des adaptations musculaires isocinétiques. Movement \& Sport Sciences - Science \& Motricité, 2013, pp.23-36. 10.1051/sm/2013074 . hal-03472406

\author{
HAL Id: hal-03472406 \\ https://hal.science/hal-03472406
}

Submitted on 9 Dec 2021

HAL is a multi-disciplinary open access archive for the deposit and dissemination of scientific research documents, whether they are published or not. The documents may come from teaching and research institutions in France or abroad, or from public or private research centers.
L'archive ouverte pluridisciplinaire HAL, est destinée au dépôt et à la diffusion de documents scientifiques de niveau recherche, publiés ou non, émanant des établissements d'enseignement et de recherche français ou étrangers, des laboratoires publics ou privés. 


\title{
Approche méthodologique et application populationnelle des adaptations musculaires isocinétiques
}

\author{
Pierre Louis Bernard ${ }^{1}$, Hubert Blain ${ }^{1,2}$, Guillaume Tallon ${ }^{1,2}$ et Sofiane Ramdani ${ }^{1}$ \\ 1 Movement to Health (M2H), Euromov, Université Montpellier 1, 700 avenue du Pic Saint Loup, \\ 34090 Montpellier, France \\ 2 Département de Médecine Interne et de Gériatrie, Centre Antonin Balmes, Centre Hospitalier Universitaire, \\ 34090 Montpellier, France
}

Reçu le 16 novembre 2012 - Accepté le 26 novembre 2012

\begin{abstract}
Résumé. L'analyse de la validité des démarches et des variables de caractérisation des adaptations musculaires isocinétiques sert la qualité de la prise en charge de la personne. D'un point de vue méthodologique, nous présentons des travaux sur la validité du paramètre de temps de développement de la force maximale (TDFM) (étude 1) et sur l'influence de trois temps de récupération de 30, 60 et 180 secondes sur les niveaux de force externe développée (étude 2). $\mathrm{Au}$ regard de son trop faible niveau de reproductibilité (CCI de 0,51-0,65 en extension et entre 0,50-0,63 en flexion), le TDFM ne peut servir fiablement la caractérisation des adaptations des muscles fléchisseurs et extenseurs du genou. Pour l'étude 2 et l'analyse des interactions entre la période de test et les trois temps de récupération, nous observons une absence systématique de différence significative entre les mesures isocinétiques de force maximale et de puissance moyenne obtenues sur les quadriceps et les ischio-jambiers. Ces données questionnent l'approche strictement énergétique et incitent à l'étude des interactions entre la production de force et les phénomènes de fatigue en relation avec les temps de récupération. Les méthodologies isocinétiques servent aussi la caractérisation des adaptations à moyen et à long termes suite à des programmes d'intervention en activités physiques. Chez la personne âgée, des résultats préliminaires témoignent des phénomènes d'optimisation et de déconditionnement de la fonction musculaire de femmes initialement sédentaires (étude 3).
\end{abstract}

Mots clés : Isocinétisme, reproductibilité, récupération, vieillissement, activité physique, genou

\begin{abstract}
Methodological approach and popilational application of isokinetic muscular adaptations.

The analysis of the validity of the methods of characterization dealing with the isokinetic muscular adaptations favoured the quality of the evaluation and the relevance of the rehabilitation program. In methodologic point of view, we analyzed the reliability of time to peak torque (TPT) (study 1) and the influence of three counter-balanced rest intervals of 30,60 and 180 seconds for isokinetic contractions of flexor-extensor knee muscles (study 2). Due to the low reproducibility (ICC between 0.51-0.65 in extension and between 0.50-0.63 in flexion), the TPT cannot serve as independent isokinetic parameters of knee flexor and extensor performance and the characterization of muscular adaptations in athletes. The analysis of the time and rest interval interaction demonstrated a systematic lack of significant difference between the isokinetic measures obtained after the counterbalanced rest intervals for peak torque and mean power of the quadriceps and of the hamstrings. These findings raised questions about a strict energetic approach and encourage future research for analyzing the interaction of force production and fatigue phenomena in relation to the rest interval. Isokinetic methodology favoured also the characterization of the middle and the term adaptations after a program of physical activities. For elderly people, a preliminary data showed the phenomenous of optimisation and detraining of the muscular function of sedentary women (study 3).
\end{abstract}

Key words: Isokinetic, reliability, recovery, ageing, physical activity, knee 


\section{Introduction}

Si l'on retrouve une valorisation et une fascination pour la force dès l'Antiquité, il nous faut attendre le milieu $\mathrm{du} \mathrm{XX}^{\mathrm{e}}$ siècle pour bénéficier des premières recherches scientifiques sur le renforcement musculaire. Aujourd'hui, à des fins de performance, d'efficience motrice ou de santé, diverses méthodologies d'évaluation et de travail musculaire co-existent.

À la fin des années 1960, le concept d'exercice musculaire isocinétique est introduit par Hislop et Perrine (1967). Ils proposent de contrôler la vitesse d'exécution du mouvement en imposant une résistance à la contraction à tous les angles de l'amplitude articulaire. Grâce à une résistance auto-adaptée, une contraction musculaire maximale est développée, à vitesse constante, sur une amplitude choisie d'une articulation du squelette appendiculaire ou du segment tronculaire. Aujourd'hui, l'évaluation est au service de la programmation des contenus nécessaires à l'optimisation de la fonction neuromotrice et musculaire. La réalisation des programmes de renforcement peut se faire sur dynamomètre pour un travail analytique à dominante neuro-musculaire et le plus souvent, couplé à un travail moteur global plurisegmentaire et à dominante énergétique.

Si l'isocinétisme peut être considéré comme un travail spécifique et «anti-physiologique », car à vitesse constante, c'est-à-dire l'inverse des mouvements réalisés par le corps humain poly-articulé, il n'en demeure pas moins qu'il offre d'une part, la maîtrise des variables de modes de contraction, d'amplitude, d'angle et de vitesse de travail, de gestion des charges de travail et des temps de récupération. Il permet d'autre part, la mesure des forces résultantes à court terme, la quantification des niveaux de récupération intra et inter-séances et la détermination des adaptations à moyen terme.

Néanmoins, dans une approche critique et méthodologique, il s'avère encore nécessaire de travailler à la définition des meilleurs protocoles d'évaluation et d'analyse scientifique par la mise en évidence de la validité des démarches et des variables de caractérisation. La maîtrise de ces démarches d'évaluation et d'analyse sert l'individualisation de l'accompagnement ou la prise en charge de la personne.

En trois temps, cet article tente d'illustrer des travaux sur la validité du paramètre de temps de développement de la force maximale (étude 1), l'influence des temps de récupération sur les niveaux de force externe développée (étude 2) et enfin, l'usage de l'isocinétisme pour la caractérisation des gains de force puis du déconditionnement de personnes âgées initialement sédentaires (étude 3).

\section{Reproductibilité des paramètres de temps de développement de la force maximale isocinétique du genou de jeunes sportifs}

La majorité des études portant sur la fonction musculaire a montré un bon niveau de reproductibilité des paramètres isocinétiques de force maximale, de travail et de puissance moyenne (Bohannon \& Smith, 1989; Kannus, 1994; Impillizzeri, Bizzini, Rampinini, Cereda, \& Maffiuletti, 2008; Julia, et al., 2010; Saenz, et al., 2010). Il existe un grand nombre de paramètres de caractérisation des adaptations musculaires mais tous ont, initialement, dû répondre de leurs critères de validation méthodologique afin de témoigner, par la suite, de leur intérêt clinique (Casartelli, Müller, \& Maffiuletti, 2010; Negrete, et al., 2010).

Depuis des années, le paramètre de temps de développement de la force maximale (TDFM) demeure controversé (Madsen, 1996; Caprinaca, Battenti, Demarie, \& Figura, 1998; Ostenberg \& Roos, 2000; Soderman, Alfredson, Pietilä, \& Werner, 2001). Il caractérise « l'explosivité musculaire » (Kannus, 1994) et quelques études ont rapporté des relations entre les vitesses angulaires mesurées au niveau articulaire et un certain nombre de dysfonctionnements musculo-articulaires (Fleisig, Andrews, Dillman, \& Escamilla, 1995), ce qui conditionnerait une partie de son intérêt. Aussi, notre objectif était de tester la reproductibilité du TDFM chez de jeunes sportifs, lors de mouvements de flexion et d'extension du genou réalisés sur les côtés dominant et nondominant à 60 et $180^{\circ} / \mathrm{s}$ (Bernard, et al., 2012).

\subsection{Méthode}

\subsubsection{Population}

La population étudiée comprenait 29 sportifs masculins pratiquant le football, la gymnastique et la natation à des niveaux de compétitions nationales (Tab. 1). Tous les participants étaient sélectionnés sur la base des critères suivants : un investissement de plus de trois ans dans leur sport respectif avec un entraînement minimum de trois séances hebdomadaires; un âge compris entre 15 et 30 ans inclus. Nous avons exclu les sportifs présentant des pathologies au niveau des quadriceps, des ischio-jambiers ou des genoux dans les 12 mois précédents les tests.

\subsubsection{Matériel et protocole expérimental}

Les tests étaient réalisés sur Cybex Orthotron $K \mathrm{KT}^{\circledR} \mathrm{du}$ centre médical hélio-marin de Vallauris. Le logiciel Humac Médimex ${ }^{\circledR}$ Windows a servi le recueil des données. Les tests étaient réalisés bilatéralement en position assise avec un angle de flexion de hanche de $110^{\circ}$, un sanglage du tronc et de la taille et les membres supérieurs croisés sur le tronc. L'amplitude de mouvement était de $90^{\circ}$ avec un premier test du côté dominant sans correction des effets de la gravité. Après dix minutes sur bicyclette ergométrique, l'échauffement était suivi de quatre contractions des extenseurs et des fléchisseurs à chacune des deux vitesses de tests sélectionnées de 60 et $180^{\circ} / \mathrm{s}$.

Le protocole expérimental comprenait trois sessions de tests isocinétiques à 60 et $180^{\circ} / \mathrm{s}$ avec un temps de 

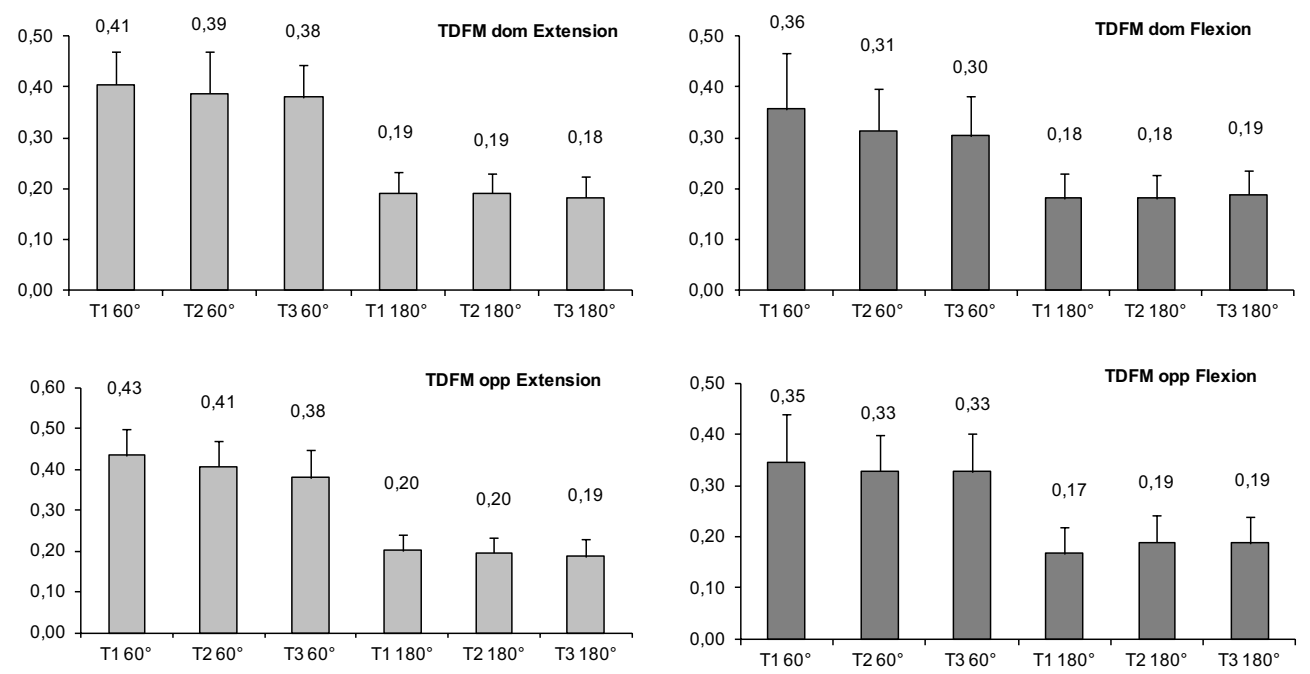

Fig. 1. Moyennes et écarts types du temps de développement de la force maximale (TDFM) lors des mouvements d'extension et de flexion des côtés dominant (dom) et opposé (opp) à $60^{\circ} / \mathrm{s}$ et $180^{\circ} / \mathrm{s}$.

Tableau 1. Caractéristiques de la population.

\begin{tabular}{ccccc}
\hline Population & & Age & Taille & Poids \\
\hline & $\mathrm{m}$ & 21.91 & 177.93 & 73.48 \\
& $\mathrm{sd}$ & 2.29 & 6.69 & 6.58 \\
\hline & & âge & taille & poids \\
\hline Footballeur & $\mathrm{m}$ & 21.33 & 178.40 & 73.13 \\
$\mathrm{n}=15$ & $\mathrm{sd}$ & 2.01 & 5.88 & 4.39 \\
\hline Gymnaste & $\mathrm{m}$ & 22.36 & 174.43 & 70.14 \\
$\mathrm{n}=7$ & $\mathrm{sd}$ & 2.11 & 7.11 & 6.28 \\
\hline Nageur & $\mathrm{m}$ & 22.71 & 180.43 & 77.57 \\
$\mathrm{n}=7$ & $\mathrm{sd}$ & 2.96 & 7.46 & 9.20 \\
\hline
\end{tabular}

récupération de 45 minutes entre chacune. Chaque test comprenait quatre contractions concentriques maximales des extenseurs et des fléchisseurs du genou sur le côté dominant à $60^{\circ} / \mathrm{s}$ puis du côté non-dominant après trois minutes de récupération. Les participants bénéficiaient alors de cinq minutes de récupération avant la seconde partie du test, composée de quatre contractions concentriques maximales des extenseurs et des fléchisseurs du genou, sur le côté dominant à $180^{\circ}$ / s puis du côté non-dominant après trois minutes de récupération.

\subsubsection{Analyse statistique}

La normalité des caractéristiques des sujets a été vérifiée par le test de Kolmogorov-Smirnov. La reproductibilité absolue, définie comme le degré de variation de mesures répétées pour un même individu, est exprimée par le paramètre d'erreur standard de mesure (SEM). Le SEM représente le pourcentage de la valeur moyenne obtenue (SEM $[\%]=$ SEM $[\mathrm{Nm}] /$ valeur moyenne des sessions). Un intervalle de confiance à $95 \%$ pour la détermination des changements minimaux détectables était calculé par la formule : $\pm 1,96 \times \sqrt{ } 2 \times \mathrm{SEM}$. Le niveau de significativité
Tableau 2. Valeurs d'erreur standard (SEM) et de coefficient de variation (CV) du temps de développement de la force maximale (TDFM) lors des mouvements de flexion et d'extension des cotés dominant et non-dominant à $60^{\circ} / \mathrm{s}$ et $180^{\circ} / \mathrm{s}$.

\begin{tabular}{ccc}
\hline TDFM & \% SEM & \% CV \\
\hline Dom Ext 60 & 0,12 & 9,8 \\
Dom Ext 180 & 0,15 & 11,1 \\
Ndom Ext 60 & 0,08 & 8,1 \\
Ndom Ext 180 & 0,13 & 10,3 \\
Dom Fl 60 & 0,18 & 16,1 \\
Dom Fl 180 & 0,16 & 14 \\
Ndom Fl 60 & 0,15 & 13 \\
Ndom Fl 180 & 0,15 & 14,7 \\
\hline
\end{tabular}

d'un $p<0,05$ était retenu. Ces analyses étaient réalisées par le logiciel Statview ${ }^{\circledR}$ software (Version 5.0, SAS Institute, Inc., Cary, NC). Pour le TDFM, la reproductibilité était testée par les coefficients de corrélation intra-classes (CCI) qui constituent des mesures relatives de reproductibilité. Ces coefficients sont des rapports de variances calculés avec des mesures répétées de type ANOVA. La valeur du CCI s'étend de 0 à 1,0 indiquant une absence de reproductibilité et 1 correspondant au niveau maximal de reproductibilité. Les valeurs de CCI étaient considérées comme excellentes si $>0,9$ et de bons niveaux entre 0,70,9 . Afin d'obtenir une analyse la plus complète possible de la reproductibilité du TDFM, nous avons calculé six types de CCI (Shrout \& Fleiss, 1979) en utilisant le logiciel Matlab ${ }^{\circledR}$.

\subsection{Résultats}

Les valeurs de coefficient de variation $(\mathrm{CV})$ et de SEM sont présentées dans le tableau 2. La figure 1 présente les valeurs isocinétiques aux deux vitesses de test. 
Tableau 3. Valeurs des coefficients de correlation intra-classes (CCI) du temps de développement de la force maximale (TDFM) mesurées durant les mouvements de flexion et d'extension des cotés dominant et non-dominant à $60^{\circ} / \mathrm{s}$ et $180^{\circ} / \mathrm{s}$.

\begin{tabular}{ccccc}
\hline EXT & TDFM dom 60 & TDFM nd 60 & TDFM dom 180 & TDFM nd 180 \\
\hline 1,1 & 0,65 & 0,57 & 0,51 & 0,57 \\
$1, \mathrm{k}$ & 0,85 & 0,80 & 0,76 & 0,80 \\
2,1 & 0,65 & 0,59 & 0,51 & 0,58 \\
$2, \mathrm{k}$ & 0,85 & 0,81 & 0,76 & 0,80 \\
3,1 & 0,66 & 0,68 & 0,51 & 0,59 \\
$3, \mathrm{k}$ & 0,86 & 0,86 & 0,75 & 0,81 \\
\hline FL & TDFM dom 60 & TDFM nd 60 & TDFM dom 180 & TDFM nd 180 \\
\hline 1,1 & 0,50 & 0,62 & 0,63 & 0,63 \\
$1, \mathrm{k}$ & 0,75 & 0,83 & 0,83 & 0,84 \\
2,1 & 0,51 & 0,62 & 0,63 & 0,64 \\
$2, \mathrm{k}$ & 0,76 & 0,83 & 0,83 & 0,84 \\
3,1 & 0,55 & 0,62 & 0,62 & 0,67 \\
$3, \mathrm{k}$ & 0,78 & 0,83 & 0,83 & 0,86 \\
\hline
\end{tabular}

En extension, du côté dominant à $60^{\circ} / \mathrm{s}$, l'usage des six méthodes d'analyse fournit des valeurs de CCI comprises entre 0,65 et 0,86 . Elles sont inférieures et comprises entre 0,51 et 0,76 à $180^{\circ} / \mathrm{s}$ (Tab. 3). Du côté nondominant, les valeurs de CCI sont comprises entre 0,57 et 0,86 à $60^{\circ} / \mathrm{s}$ et entre 0,57 et 0,81 à $180^{\circ} / \mathrm{s}$ (Tab. 3 ).

En flexion, du côté dominant à $60^{\circ} / \mathrm{s}$, nous observons des valeurs de CCI comprises entre 0,50 et 0,78 et à $180^{\circ} / \mathrm{s}$, entre 0,63 et 0,83 (Tab. 3). Du côté non-dominant, les valeurs de CCI sont situées entre 0,62 et 0,83 à $60^{\circ} / \mathrm{s}$ et entre 0,63 et 0,86 à $180^{\circ} / \mathrm{s}$ (Tab. 3).

\subsection{Discussion}

L'analyse globale permet de qualifier d'insuffisant le niveau de reproductibilité du paramètre de TDFM testé chez une population de jeunes sportifs. La méthode d'analyse s'appuie sur six modes de calcul plus ou moins exigeants et fournit donc des valeurs échelonnées. La valeur de reroductibilité, le plus souvent positionnée entre 0,60 et 0,80 , nécessite une analyse par paramètre et par groupe musculaire testés. Le recours au CV et aux SEM complète l'analyse.

Chez ces sportifs, la reproductibilité du TDFM des quadriceps est supérieure à 60 qu'à $180^{\circ} / \mathrm{s}$. Elle atteint 0,86 tant du côté dominant qu'opposé avec une valeur basale à 0,57 , ce qui incite à considérer ce niveau de reproductibilité comme insatisfaisant. À $180^{\circ} / \mathrm{s}$, la reproductibilité est d'un niveau plus faible, ce qui est envisageable si l'on considère que la résistance au mouvement est inférieure à vitesse rapide, ce qui favorise plus tardivement la rencontre de celle-ci et que d'autre part, le travail strictement isocinétique est moins long. De plus, nous pouvons émettre l'hypothèse que la typologie musculaire à dominante lente du groupe quadricipital puisse influencer un meilleur niveau de reproductibilité à une plus faible vitesse de travail. Quels que soient les justificatifs, nous considérerons donc que pour ce groupe musculaire, les meilleures conditions méthodologiques sont réunies à une vitesse d'évaluation plus lente. En ce qui concerne les ischio-jambiers, fléchisseurs du genou, les niveaux de reproductibilité semblent être à l'inverse des quadriceps, légèrement meilleurs à la vitesse rapide de $180^{\circ}$ / s avec des valeurs proches des deux côtés controlatéraux et situées entre 0,62 et 0,86 . Ils sont donc moins bons à vitesse lente en s'échelonnant entre 0,50 à $60^{\circ} / \mathrm{s}$ du côté dominant pour le mode de calcul le plus critique et 0,83 du côté opposé pour le mode le plus tolérant.

En synthèse, nous considérerons que le TDFM est un paramètre dont le niveau de reproductibilité est insuffisant pour participer à la caractérisation des adaptations musculaires du sportif. Ce trop faible niveau de reproductibilité confirme les travaux réalisés chez des sujets sains (Croisier \& Crielaard, 1999) et chez un groupe de 24 jeunes femmes (Madsen, 1996) et est donc en contradiction avec les travaux mettant en évidence une reproductibilité satisfaisante chez un groupe de 18 femmes âgées (Caprinaca, et al., 1998) et un groupe de 178 sujets âgés de 45 à 78 ans (Frontera, Hugues, Dallal, \& Evans, 1993).

À titre clinique, mais limité d'un point de vue méthodologique comme nous venons de le mettre en évidence, ce paramètre de TDFM est classiquement retenu dans l'identification des qualités physiques du sportif. Il peut être assimilé à une des composantes de la force qu'est l'explosivité. De part leur validation et ce qu'ils représentent, les paramètres de force maximale et de travail caractérisent classiquement les capacités fonctionnelles tandis que les variables de nature d'exercice, d'intensité, de durée et de fréquence complètent la caractérisation des charges d'entraînement et des comportements individuels. Avec de tels niveaux de reproductibilité obtenus par les calculs d'ICC, il est nécessaire de rester critique sur l'intégration du TDFM à l'objectivation des qualités musculaires individuelles. Les valeurs de CV comprises entre 8,1 et 16,1 \% et supérieures à $10 \%$, dans six des huit conditions de tests (Tab. 2), ainsi que les valeurs de SEM globalement proches de $0,15 \%$ confirment le niveau méthodologique insuffisant. Plus encore, il faut être critique quant à la validité du TDFM à servir l'identification d'éventuelles causes de 
dysfonctionnements en relation avec la capacité à créer une brusque variation de vitesse. Cette accélération créée et subie au niveau musculo-articulaire nécessite tout à la fois, l'intégrité des structures péri-articulaires assurant un travail physiologique et un contrôle nerveux du mouvement qui va idéalement de pair avec l'efficience motrice. Les notions de sens de la position et du mouvement qui mobilisent la proprioception et le traitement des informations d'origine kinesthésique participent à cette régulation.

Nous considérerons que le TDFM est une valeur limitée dans la recherche de compréhension et le contrôle des adaptations afin de servir la programmation de l'entraînement intégrant l'optimisation et la prévention. Cette étude comporte certaines limites qui justifieraient d'adaptations relatives au nombre restreint des vitesses de test et aux profils sportifs et hétérogènes des populations testées. Il serait pertinent de définir précisément, l'influence d'une pratique sportive sur la reproductibilité des valeurs de force développée ainsi que les réponses de personnes âgées ou de personnes en phase de réhabilitation.

\subsection{Conclusion}

Nos résultats indiquent que les valeurs de reproductibilité du TDFM ne sont pas suffisantes pour caractériser les adaptations musculaires des fléchisseurs et des extenseurs du genou de sportifs. Au regard des faibles niveaux de reproductibilité obtenus par le calcul des CCI, de même que par le SEM et le CV, un usage limité du TDFM est recommandé pour caractériser les qualités musculaires individuelles.

\section{Influence du temps de récupération sur la force maximale isocinétique du genou d'hommes sains}

\subsection{Introduction}

Dans le domaine de l'exercice musculaire, de nombreux facteurs métaboliques et neuro-physiologiques sont à prendre en compte pour favoriser l'expression des capacités de production de force. Au sein des méthodologies de programmation, la gestion des volumes et de l'intensité des exercices conditionne à la fois, la réalisation de performance (Baechle, 2008), la poursuite de programme de renforcement musculaire et de réhabilitation et la prévention de dysfonctionnements musculo-articulaires potentiellement dus à une planification inadaptée.

Aujourd'hui, dans une approche rationnelle de l'entraînement et de l'évaluation, diverses données scientifiques influencent directement les méthodologies de programmation et la gestion des temps de travail et de récupération et justifient de nombreux travaux. Ce besoin d'articulation se pose dans la définition des délais entre les sessions de travail au sein d'un cycle; il en est de même à court terme, au sein d'une session d'entraînement ou d'évaluation où les unités varient et s'expriment alors en secondes ou en minutes. Il est connu qu'un temps de récupération optimal est nécessaire entre des efforts pour diminuer les phénomènes de fatigue musculaire périphérique et faciliter la récupération au sein du muscle de part les facteurs de remise en cause de production de force tels que l'accumulation d'ions $\mathrm{H}^{+}$, de phosphate inorganique, la déplétion des réserves en créatine phosphate $(\mathrm{CP})$, des perturbations du couplage excitation-contraction (Lindinger, Mc Kelvie, \& Heigenhauser, 1995; Westerblad, Allen, Bruton, Andrade, \& Lännergren, 1998; Forbes, Raymer, Kowalchuk, Thompson, \& Marsh, 2008; Machado, Katch, \& Katch, 2011).

D'un point de vue énergétique, il est connu que les concentrations intramusculaires en $\mathrm{CP}$ sont faibles (18 à $20 \mathrm{mmol} \cdot \mathrm{kg}^{-1}$ de poids frais) et que la déplétion de ce substrat énergétique de liaisons phosphates à haut potentiel énergétique est immédiate. Aussi, afin de justifier des plus hauts niveaux de production de force, l'instauration d'un temps de récupération est rapidement nécessaire, sous peine de mobiliser des substrats énergétiques dépendant des glycolyses anaérobie puis aérobie conduisant à une diminution des forces développées. L'ensemble des ouvrages de référence en physiologie de l'exercice considère que la réplétion de la $\mathrm{CP}$ s'effectue dans les cinq minutes suivant l'arrêt de l'effort et qu'un apport suffisant en oxygène favorise cette resynthèse (Mc Ardle, Katch, \& Katch, 2006; Wilmore, Costill, \& Kenney, 2008). D'un point de vue plus strictement énergétique, il serait logique de considérer que suite à un effort de dix secondes de contractions maximales continues, la déplétion en $\mathrm{CP}$ serait quasi totale et qu'un temps de récupération de l'ordre de trois minutes, favoriserait une meilleure resynthèse et par conséquent, une force maximale plus élevée, lors d'un second effort, qu'avec un temps de récupération égal à une minute.

L'observation des protocoles d'évaluation isocinétiques rassemble des temps de récupération (TR) extrêmement variables, allant de quelques secondes à plusieurs minutes. Divers travaux évoquent que la diminution des temps de récupération entre des séries, au sein de protocole de renforcement musculaire de plusieurs semaines, serait un facteur favorisant la production d'une force supérieure. Ces données sont rapportées lors d'un protocole en mode isotonique sur les muscles biceps brachii (Rooney, Herbert, \& Balnave, 1994) et lors d'un travail comparant une vs. deux minutes de récupération sur le gain de force du quadriceps suite à un réentraînement isométrique (Schott, Cully, \& Rutherford, 1995). Bottaro, Russo, \& Oliveira (2005) considèrent que 30 secondes de récupération sont suffisantes pour récupérer le niveau de force maximal chez 20 sujets âgés sédentaires et Parcell, Sawyer, Tricoli, \& Chinevere (2002) concluent que 60 secondes de récupération sont suffisantes pour récupérer le niveau de force maximal 
d'extension du genou chez de jeunes adultes sains. À l'inverse, Robinson, et al., (1995) observent une augmentation significativement supérieure de la force maximale développée en squat, suite à l'application d'un protocole de renforcement musculaire utilisant trois minutes vs. 30 secondes de récupération. Pincivero, Lephart, \& Karunakara (1998) montrent une réduction notable de la force maximale d'extension du genou à $90^{\circ} / \mathrm{s}$ avec 40 vs. 160 secondes de récupération entre les séries chez 15 adolescents. Touey, Sforzo, \& Mc Manys (1994) testant 28 jeunes hommes, rapportent une diminution significative de force isocinétique maximale d'extension du genou à 60 ou $180^{\circ} / \mathrm{s}$ réalisés avec 30 et 60 secondes de récupération entre les séries. En plus des aspects énergétiques, la recherche des effets du rapport temps de travail/temps de récupération permet d'introduire des notions neuro-musculaires telle que la potentialisation se manifestant par une augmentation de la force développée après une activité musculaire volontaire ou évoquée. Elle constitue une justification potentielle, ou tout du moins une hypothèse à l'absence d'effet de certains temps de récupération appliqués à diverses populations au sein de protocole utilisés.

L'objectif de notre travail était de définir l'influence du temps de récupération sur la force musculaire isocinétique concentrique développée au niveau du couple extenseurs/fléchisseurs du genou du côté dominant (Bernard, et al., 2013). Les trois temps de récupération de 30, 60 et 180 secondes étaient positionnés aléatoirement, au sein de trois séances et appliqués après un premier test au sein de chaque séance d'évaluation.

\subsection{Méthode}

\subsubsection{Population}

14 hommes composaient la population d'étude. Les valeurs moyennes et d'écart type pour l'âge, le poids et la taille étaient respectivement de 32,5 ans $( \pm 8)$, $74,2 \mathrm{~kg}( \pm 10,3)$ et $177,5 \mathrm{~cm}( \pm 8,2)$. Les critères de non-inclusion étaient relatifs à la pratique de sports de compétition et d'entraînement intensif durant la période des tests, la présence de pathologies chroniques ou aiguës du genou et/ou des membres inférieurs, des antécédents de chirurgie du genou, des douleurs du genou et/ou des membres inférieurs, une pathologie cardio-vasculaire contre-indiquant la pratique d'activité physique et sportive.

\subsubsection{Protocole de test}

Le protocole général de l'étude comprenait trois tests isocinétiques du genou du côté dominant à 48 heures d'intervalle. La même procédure d'échauffement et d'installation du sujet était systématiquement reproduite considérant que l'objectif de l'étude était de tester l'influence de trois temps de récupération différents appliqués de manière aléatoire. Chacune des trois sessions d'évaluation débutait donc par un échauffement, l'application d'un premier test suivi d'un temps de récupération tiré aléatoirement et d'une seconde épreuve isocinétique.

Avant l'évaluation, chaque sujet bénéficiait d'une période d'échauffement sur bicyclette ergométrique durant cinq minutes à une résistance de 60 watts et à 90 rotations par minute. Ce travail dynamique était suivi de mouvements d'étirement des muscles quadriceps et ischio-jambiers. Les sujets ont été évalués sur dynamomètre isocinétique Biodex Système $3{ }^{\circledR}$ avec correction de la gravité. L'évaluation a été réalisée en mode concentrique/concentrique sur une amplitude articulaire de $90^{\circ}$ avec placement de l'appui jambier à deux doigts sus-maléolaire et des butées placées respectivement à $-10^{\circ}$ en retrait de la verticale et $80^{\circ}$ d'extension dans le plan antérieur afin de limiter la résistance des ischio-jambiers au travail d'extension du genou. L'évaluation des muscles fléchisseurs et extenseurs du genou a été réalisée avec un sanglage du tronc et de la cuisse active, un angle de $110^{\circ}$ entre le tronc et les cuisses et les membres supérieurs croisés sur le tronc afin de limiter les compensations. Chaque personne était placée dans une position confortable ne limitant pas les mouvements du genou. La hauteur et la profondeur de l'assise par rapport à l'axe de rotation du système et la longueur du bras de levier par rapport à l'axe de rotation étaient mémorisées dans le programme informatique (Biodex Medical Inc.) afin de standardiser les positions de tests de chaque sujet et de les appliquer à chacune des trois sessions de tests. Après installation sur le siège d'évaluation, chaque sujet était soumis à un échauffement standardisé de deux séries de cinq répétitions à $180^{\circ} / \mathrm{s}$ séparées d'une minute de récupération. Une période de trois minutes espaçait la fin de l'échauffement de l'application du premier test.

Le protocole d'évaluation débutait par une série de cinq répétitions à $90^{\circ} / \mathrm{s}$. S'appliquait alors un des trois temps de récupération de 30,60 ou 180 secondes, tiré aléatoirement par l'expérimentateur au début de l'étude. Les paramètres de pic de couple (PC) (N.m) et de puissance moyenne (PM) (W) ont été retenus pour l'analyse des résultats. Au cours du test, chaque sujet bénéficiait, systématiquement, des mêmes encouragements verbaux exprimés par le même expérimentateur responsable de l'étude.

\subsubsection{Analyse statistique}

Après vérification de la normalité de la distribution, les valeurs moyennes et de déviation standard ont été calculées pour l'ensemble des variables. Pour les paramètres de $\mathrm{PC}$ et de PM, le SEM en valeur absolue et exprimé en pourcentage, le CV (\%) et le calcul du coefficient de corrélation intra-classe ont été calculés à partir des valeurs du premier test de chacune des trois sessions. 
Tableau 4. Valeurs moyennes et d'écart type de force maximale (PC) et de puissance (PM) des muscles Quadriceps (Q) et Ischio-jambiers (IJ) mesurées lors du test initial puis suite à l'application du temps de récupération de 30 secondes (30 REC), 60 secondes (60 REC) ou 180 secondes (180 REC).

\begin{tabular}{ccccccccc}
\hline 30 REC & PC Q & PC Q 30 & PC IJ & PC IJ 30 & PM Q & PM Q 30 & PM IJ & PM IJ 30 \\
\hline $\mathrm{m}$ & 240.1 & 241.8 & 129.2 & 121.8 & 148.4 & 150.5 & 90.1 & 86.9 \\
$\mathrm{sd}$ & 43.1 & 41.5 & 23.7 & 22.8 & 34.0 & 30.5 & 20.4 & 18.2 \\
\hline $60 \mathrm{REC}$ & PC Q & PC Q 60 & PC IJ & PC IJ 60 & PM Q & PM Q 60 & PM IJ & PM IJ 60 \\
\hline $\mathrm{m}$ & 242.1 & 245.8 & 130.3 & 127.8 & 149.6 & 150.0 & 95.4 & 89.6 \\
$\mathrm{sd}$ & 50.9 & 53.6 & 25.0 & 28.9 & 35.5 & 39.0 & 18.9 & 23.9 \\
\hline $180 \mathrm{REC}$ & PC Q & PC Q 180 & PC IJ & PC IJ 180 & PM Q & PM Q 180 & PM IJ & PM IJ 180 \\
\hline $\mathrm{m}$ & 238.6 & 242.3 & 140.7 & 140.7 & 147.6 & 151.3 & 97.9 & 98.5 \\
$\mathrm{sd}$ & 59.3 & 58.9 & 29.1 & 30.8 & 39.4 & 38.9 & 23.7 & 19.6 \\
\hline
\end{tabular}

L'analyse de l'influence du temps de récupération sur les valeurs de force a été réalisée par une analyse de variance à deux facteurs (test $\times$ temps de récupération). Un niveau de significativité de $p<0,05$ a systématiquement été retenu pour l'ensemble des analyses.

\subsection{Résultats}

Ce travail justifie d'un premier axe d'analyse à dominante méthodologique concernant l'analyse de l'effet temps. La comparaison des valeurs mesurées lors de l'épreuve isocinétique initiale de chacune des trois sessions d'évaluation démontre une absence de différence significative $(0,54<p<0,79)$. Les analyses de l'erreur standard, du coefficient de variation et du coefficient de corrélation intra-classe $(0,75<\mathrm{ICC}<0,91)$ complètent cette observation et témoignent du bon niveau de reproductibilité. Le tableau 4 synthétise les valeurs moyennes et d'écarts types de force maximale et de puissance moyenne du groupe de 14 sujets sains testés au début de chaque session d'évaluation, puis après un des trois temps de récupération, aléatoire de 30,60 ou 180 secondes.

Le tableau 5 présente les valeurs statistiques obtenues pour chacun des facteurs période $(\mathrm{P})$ et temps de récupération (Recup) et pour l'interaction entre ces deux facteurs $(\mathrm{R} \times \mathrm{P})$. Une absence de différence significative est systématiquement observée pour les interactions R-Ppour le couple maximal des quadriceps $(F=0,0034, p=$ $0,99)$, le couple maximal des ischio-jambiers $(F=0,13, p$ $=0,88)$, la puissance moyenne des quadriceps $(F=0,013$, $p=0,98)$ et la puissance moyenne des ischio-jambiers $(F$ $=0,15, p=0,86$ ) (Tab. 5). Pour ce qui concerne les effets simples, nous n'observons aucune influence significative du temps de récupération sur les valeurs de force et de puissance développées (Tab. 5). Pour l'effet temps, l'absence de différence significative était attendue et s'observe à la fois pour le couple maximal des quadriceps et des ischio-jambiers ainsi que pour les puissances moyennes de ces deux groupes musculaires.
Tableau 5. Valeurs du test d'analyse de variance et niveau de significativité pour le facteur de temps de récupération (Recup), le facteur Période (P) et l'interaction des deux facteurs pour les paramètres de Pic de Couple (PC) des quadriceps (Quadri) et des ischio-jambiers (ischio) et de Puissance Moyenne (PM).

\begin{tabular}{cccc}
\hline & & $\mathrm{F}$ & $\mathrm{P}$ \\
\hline PC Quadri & Recup & 0.0373 & 0.96 \\
& $\mathrm{P}$ & 0.0686 & 0.79 \\
& $\mathrm{R} \times \mathrm{P}$ & 0.00345 & 0.99 \\
\hline PC Ischio & Recup & 2.252 & 0.11 \\
& $\mathrm{P}$ & 0.305 & 0.58 \\
& $\mathrm{R} \times \mathrm{P}$ & 0.13 & 0.87 \\
\hline PM Quadri & Recup & 0.000857 & 0.99 \\
& $\mathrm{P}$ & 0.0662 & 0.79 \\
& $\mathrm{R} \times \mathrm{P}$ & 0.0137 & 0.98 \\
\hline PM Ischio & $\mathrm{Recup}$ & 1.401 & 0.25 \\
& $\mathrm{P}$ & 0.365 & 0.54 \\
& $\mathrm{R} \times \mathrm{P}$ & 0.149 & 0.86 \\
\hline
\end{tabular}

\subsection{Discussion}

Ce travail justifie d'un premier axe d'interprétation à dominante méthodologique concernant l'analyse de l'effet temps. La comparaison des valeurs mesurées lors de l'épreuve isocinétique initiale de chacune des trois sessions d'évaluation démontre une absence de différence significative $(0,55<p<0,80)$ (Tab. 5 ). Les analyses de l'erreur standard, du coefficient de variation et du coefficient de corrélation intra-classe $(0,75<\mathrm{ICC}<0,91)$ complétent ces données et témoignent du bon niveau de reproductibilité. Dans le domaine isocinétique, le haut niveau de reproductibilité des mesures de $\mathrm{PC}$ et de $\mathrm{PM}$ est connu et particulièrement lors de l'évaluation du genou. Il est satisfaisant de l'observer dans cette étude et de contrôler que l'état fonctionnel et le niveau de récupération interépreuve de nos sujets n'influencent pas l'étude du TR intra-épreuve.

L'analyse des interactions entre la période de test et le TR (Tab. 5) démontre une absence systématique de différence significative entre les mesures obtenues après application des trois $\mathrm{TR}$ pour le PC des quadriceps 
$(p=0,99)$, le $\mathrm{PC}$ des ischio-jambiers $(p=0,87)$, la $\mathrm{PM}$ des quadriceps $(p=0,98)$ et la PM des ischio-jambiers $(p=0,86)$. Ces données interrogent une approche strictement énergétique considérant les délais nécessaires à la resynthèse des substrats de type phosphocréatine largement mobilisés lors d'un effort intense de quelques secondes, identique à notre épreuve. Néanmoins, nos observations sont en accord avec une partie des données de la littérature qui demeure controversée en fonction des protocoles retenus tels que la vitesse de test, le nombre de séries, le nombre de mouvements ou les muscles testés, ou en fonction de facteurs relatifs aux caractéristiques de la population tels que l'âge, le sexe ou encore les niveaux d'activité ou de sédentarité des sujets. Celes, Brown, Pereira, Junior, \& Bottaro (2010) étudient les effets de deux TR de 60 et 120 secondes sur le PC du quadriceps chez 17 jeunes femmes et 16 jeunes hommes, testés sur trois séries de dix contractions unilatérales à 60 et $180^{\circ} / \mathrm{s}$ lors de deux séances. Ils concluent à l'influence significative de la vitesse de test et au rôle du sexe sur les forces développées. Bottaro, Russo \& Oliveira (2005) analysent les valeurs de 17 jeunes hommes et de 20 hommes âgés et démontrent l'influence de l'augmentation du TR (60 vs. 120 secondes) avec un PC et un travail total (TT) durant la $3^{\mathrm{e}}$ série significativement supérieurs quand deux minutes et non une minute de TR sont appliquées tant chez les jeunes que chez les personnes âgées. Theou, Gareth, \& Brown (2008) confirment l'influence de l'âge et la récupération plus rapide caractérisant les sujets plus âgés, lors d'une étude originale sur les forces des fléchisseurs et des extenseurs du genou de jeunes et de femmes âgées actives, testées durant trois séries de huit répétitions à $60^{\circ} / \mathrm{s}$. Le protocole de la présente étude est similaire à ceux observés dans la littérature et testant la force musculaire. La revue synthétique présentée dans les paragraphes ci-dessus suggère qu'un grand nombre de facteurs influence les forces développés et les effets des TR. Avec notre protocole, nous n'observons pas de diminution des forces développées avec la baisse du TR. Aussi, considérant ces travaux et nos propres résultats, et en accord avec Parcell, Sawyer, Tricoli, \& Chinevere (2002) testant l'influence de quatre TR tirés aléatoirement $(15,60,180$ et 300 s), nous considérons qu'un temps de récupération de 60 secondes est optimal pour le développement de la force maximale.

«Un facteur clé déterminant la capacité à réaliser une succession d'exercices est la longueur du TR entre les exercices »(Willardson, 2006). Cette citation renvoie au fait que l'analyse du TR n'est pas propre à l'épreuve d'évaluation mais concerne aussi les protocoles de renforcement et de réentraînement musculaire. C'est la démarche de Pincivero, Lephart, \& Karunakara (1997) qui montrent qu'un TR plus long entraîne des gains significativement supérieurs qu'un TR court au niveau des ischio-jambiers. Dans un autre travail, Pincivero et Campy (2004) étudient les effets d'un entraînement mobilisant deux TR $(40$ vs. $160 \mathrm{~s})$ sur la force du quadriceps testée après six semaines d'entraînement.
Ils démontrent une augmentation significative du PC spécifique à l'application d'un long TR lors des six semaines de renforcement. Ils n'observent pas de changement significatif de PM et de TT suite à l'entraînement et à l'application de deux TR. Ces deux études montrent l'influence des TR et insistent sur les effets différenciés des $\mathrm{TR}$ en fonction des paramètres mesurés ( $\mathrm{PC}$ vs. PM et TT) et en fonction des groupes musculaires analysés (quadriceps vs. ischio-jambiers).

L'habileté à maintenir un niveau de force peut être considérée comme un compromis entre l'altération liée à la fatigue et les stratégies neuro-musculaires permettant la réalisation d'un travail et d'une performance (Hamada, Sale, Mc Dougall, \& Tarnopolsky, 2003). Les mécanismes de potentiation et de fatigue interviennent simultanément (Rassier \& Mc Intosh, 2000) et sur un modèle assez comparable à celui évoqué pour les TR. La potentiation est dépendante à la fois, du niveau d'entraînement des sujets, du type d'effort réalisé (vitesse lente ou vitesse rapide), de l'âge des sujets qui répondent différemment à la fatigue (Behm, Anderson, \& Curnew, 2002; Ratel, Duché, \& Williams, 2006; Dipla, et al., 2009). Le rôle de la potentiation est observé par Chaouchi, et al. (2011) lors d'un effort de dix contractions isocinétiques maximales à vitesse lente $\left(60^{\circ} / \mathrm{s}\right)$ et à vitesse rapide $\left(300^{\circ} / \mathrm{s}\right)$, chez un groupe de 16 jeunes, exposés à quatre TR différenciés et randomisés. Les auteurs évoquent le rôle de la potentiation chez ce profil de sujets et son application plus spécifique aux ischio-jambiers. L'analyse de l'excitabilité musculaire (Allen, Gandevia, \& Mc Kenzie, 1995) avec la technique de twitch interpolation avant et après contraction maximale volontaire, constitue une perspective. Théoriquement, des diminutions dans de mêmes proportions de l'amplitude de l'onde $\mathrm{M}$ et de l'amplitude de la secousse évoquée indiqueraient une altération au niveau de l'excitabilité musculaire. À l'inverse, un changement au niveau de la réponse mécanique sans modification de l'onde M serait le témoin d'une altération du couplage excitation-contraction.

\subsection{Conclusion}

Ce travail confirme le haut niveau de reproductibilité des paramètres de force maximale et de puissance moyenne mesurés lors de tests isocinétiques de flexion-extension du genou. Au regard de l'objectif principal de l'étude, les résultats indiquent une absence de différence significative d'influence des trois temps de récupération situés entre 30 et 180 secondes sur les valeurs de performance musculaire. Les valeurs de force maximale et de puissance moyenne des muscles fléchisseurs et extenseurs du genou ne varient pas significativement lors de l'application des trois temps de récupération. Ce travail participe à l'analyse controversée des effets des temps de récupération. Une future étude serait nécessaire pour déterminer les adaptations neuromusculaires et les mécanismes mis en jeu durant la production de force et l'application de différents temps 
de récupération. Une telle approche permettrait d'analyser les interactions existantes entre les phénomènes de fatigue musculaire et de récupération.

\section{Entraînement et déconditionnement de la fonction musculaire de femmes âgées sédentaires et ostéopéniques}

\subsection{Introduction}

La fonction musculaire de la personne âgée est l'objet de beaucoup d'attention (Paterson, Jones, \& Rice, 2007). Que ce soit au titre de la prévention primaire des effets de l'âge afin de limiter la perte d'efficience ou au titre de la prévention secondaire afin de reconditionner et de réadapter une fonction déficiente, de multiples démarches et méthodes existent. Face à ces objectifs, l'isocinétisme constitue une méthode privilégiée d'évaluation. Nous verrons dans un premier temps, les effets de l'âge sur la fonction musculaire et l'application de l'isocinétisme à cette population. Dans un second temps, nous présenterons les résultats préliminaires d'un travail mené chez des femmes sédentaires ostéopéniques réentraînées durant six mois par de la marche active et réévaluées à 12 mois post-entraînement.

\subsubsection{Amyotrophie et sarcopénie}

Avec l'avance en âge, la baisse des capacités contractiles volontaires est causée par différents facteurs architecturaux et typologiques, neuro-musculaires et métaboliques. Cela induit une baisse plus significative des paramètres de force maximale, particulièrement sensibles à l'âge que des caractéristiques sous-maximales telles que la puissance et l'endurance musculaire.

L'amyotrophie correspond à une dégénérescence progressive qui débute dès 25 ans et connaîtra une accélération au-delà de 50 ans. À cet âge, la perte de surface musculaire est de l'ordre de $10 \%$ et pourra atteindre $50 \%$ au niveau de groupes musculaires tels le quadriceps, au-delà de 80 ans. Il est aujourd'hui classique d'utiliser le terme de sarcopénie qui renvoie non exclusivement à une perte quantitative de masse musculaire mais aussi à la dégradation de la qualité musculaire et fait référence à la surface active du muscle (surface de section transversale physiologique/SSTP). Au sein du muscle, le vieillissement s'accompagne d'une redistribution des trois principaux tissus et la diminution globale du volume musculaire ne rend qu'imparfaitement compte de l'évolution des tissus gras et conjonctifs (Paterson, et al., 2007). Ainsi, alors que le matériel contractile subit les effets de l'âge et dégénère, les deux tissus de soutien augmentent au sein du muscle (Lexell \& Downham, 1991 ; Frontera, Hughes, Fielding, Fiatarone, Evans, \& Roubenoff, 2000). Rice, Cunningham, Paterson, \& Lefcoe (1989) observent une augmentation de tissus non-musculaires des bras de $27 \%$ pour les fléchisseurs et de $45 \%$ pour les extenseurs. $\mathrm{Au}$ niveau du tronc et du rectus abdominis, la diminution de masse musculaire peut atteindre plus de $60 \%$ à 70 ans et de l'ordre de $75 \%$ à 85 ans. Pour le membre inférieur, une réduction de la surface de section transversale est observée au niveau du psoas major et du sacrospinalis (Imamura, Ashida, Ishikawa, \& Fujii, 1983), du quadriceps (Frontera, et al., 2000) et au niveau des fléchisseurs plantaires avec une augmentation des tissus non-contractiles (Macaluso, Nimmo, Foster, Cockburn, Mc Millan, \& De Vito, 2002).

De plus, la sarcopénie s'accompagne d'adaptations neurologiques et neuro-musculaires. La dégénérescence neuronale et la démyélinisation des fibres nerveuses (Kawamura, Okazaki, O’Brien, \& Dych, 1977) remettent en cause la qualité de la circulation de l'influx nerveux et le contrôle de l'activité musculaire. Les phénomènes de mort cellulaire, au niveau de la fibre musculaire ellemême mais aussi au niveau du complexe de l'unité motrice (UM) composé d'un motoneurone $\alpha$ et de l'ensemble des fibres musculaires qu'il innerve, conduisent à une diminution du nombre des unités motrices fonctionnelles (Doherty, Vandervoort \& Brown 1993; Doherty, Vandervoort, Taylor, \& Brown, 1993) et à une augmentation de la taille des unités motrices restantes de part les reconnexions des fibres musculaires libres avec les unités motrices voisines et fonctionnelles. Par l'observation de l'augmentation de l'amplitude et de durée de leurs potentiels d'action, des travaux témoignent de cet accroissement de la taille des UM (Stalberg, Borges, \& Ericsson, 1989). Le phénomène de dénervation/réinervation des fibres musculaires avec regroupement est nommé « grouping » et s'accompagne dans le même temps, d'une baisse de la capacité de bourgeonnement terminal des neurones (Mc Comas, 1996). De plus, une diminution de l'excitabilité liée à une réduction du potentiel de membrane, ainsi qu'une baisse et plus grande variabilité du taux de décharge des UM (Erim, Faisal Beg, Burke, \& De Luca, 1999; Laidlaw, Bilodeau, \& Enoka, 2000) justifient de la remise en cause de l'efficience neuro-musculaire. Cette évolution affecte préférentiellement les unités motrices de plus gros diamètres responsables des mouvements à vitesse rapide ou à intensité élevée. Les fibres de type I, c'est-à-dire « lentes » et à métabolisme oxydatif, profitent de ces dénervations en établissant de nouvelles connexions. La diminution du moment maximal est justifiée par une augmentation relative de ces fibres de types I (Lexell, 1995) et par une baisse des capacités d'activation et de contrôle de la force développée comme en témoigne la baisse du rapport moment maximal/SSTP. La baisse de l'activité électrique musculaire maximale observée tant au niveau du membre supérieur (Pousson, Lepers, \& van Hoecke, 2001) qu'au niveau du membre inférieur lors de travaux portant sur des sujets âgés, témoigne de l'atteinte nerveuse inhérente à la sarcopénie.

Bien que certaines études considèrent que la baisse des capacités puisse être minime lorque l'on rapporte la force 
Tableau 6. Valeurs de couple maximal mesurées chez des personnes âgées de genre féminin (F) et masculin (H) en condition isocinétiques concentriques et excentriques (E) et de perte de force lors de comparaison à un groupe témoin jeune (20-30 ans) (Porter, et al., 1995).

\begin{tabular}{cccccc}
\hline Étude & $\begin{array}{c}\text { Age } \\
(\mathrm{ans})\end{array}$ & Sexe & $\begin{array}{c}\text { Vitesse } \\
\left(\circ / \mathrm{s}^{-1}\right)\end{array}$ & $\begin{array}{c}\text { Couple } \\
(\mathrm{Nm})\end{array}$ & $\begin{array}{c}\text { Perte Force } \\
(\%)\end{array}$ \\
\hline Murray, et al. 1985 & $70-86$ & $\mathrm{~F}$ & 36 & 92 & 40 \\
Stalberg, et al. 1989 & $60-70$ & $\mathrm{H} . \mathrm{F}$ & 90 & $150 / 90$ & $30 / 40$ \\
Harries Bassey 1990 & 68 & $\mathrm{~F}$ & $60 / 300$ & $55 / 35$ & 30 \\
Vandervoort, et al. 1990 & $66-89$ & $\mathrm{~F}$ & $45 / 90$ & $72 / 61$ & 50 \\
& & & & $94 / 100(\mathrm{E})$ & $40 / 35(\mathrm{E})$ \\
Frontera, et al. 1991 & $65-78$ & $\mathrm{H} / \mathrm{F}$ & 60 & $144 / 89$ & 20 \\
Overend, et al. 1992 & $65-77$ & $\mathrm{H}$ & 120 & 138 & 30 \\
Poulin, et al. 1992 & $60-75$ & $\mathrm{H}$ & $90 / 180$ & $124 / 113$ & 30 \\
& & & & $160 / 183(\mathrm{E})$ & $20 / 2(\mathrm{E})$ \\
Grimby, et al. 1992 & $78-84$ & $\mathrm{H}$ & $30 / 120$ & $130 / 100$ & $\times$ \\
Stanley et Taylor 1993 & $60-70$ & $\mathrm{~F}$ & $60 / 120$ & $65 / 55$ & $\times$ \\
Porter, et al. 1995 & $62-88$ & $\mathrm{H}$ & 90 & $119 / 175(\mathrm{E})$ & $40 / 25$ \\
\hline
\end{tabular}

développée à la surface efffective de section du muscle, la majorité des études montre que quelle que soit l'articulation testée, les valeurs de force musculaire de sujets âgés sont inférieures aux jeunes dans des proportions allant de 55 à $75 \%$ pour le mouvement d'extension du genou de groupes de 70 à 90 ans, de l'ordre de $45 \%$ pour la force des doigts (handgrip) pour des hommes et des femmes de 80 à 100 ans et de l'ordre de 80 à $85 \%$ pour le mouvement de flexion du coude de personnes de 70 à 80 ans (Bernard, Seynnes, \& Blain, 2010). La mobilisation plus fréquente de l'articulation du coude dans les mouvements de préhension et de manipulation est classiquement avancée pour justifier des moindres effets de l'âge sur la décroissance des forces du membre supérieur. À l'inverse, une sédentarisation associée à l'avancé en âge entraînent une perte des capacités de contraction des muscles des membres inférieurs (extension genou/flexion plantaire et dorsale) et une remise en cause de l'autonomie locomotrice. Il en est de même pour les forces dynamiques mesurées en condition isocinétique (Porter, Myint, Kramer, \& Vandervoort, 1995), (Tab. 6). L'ensemble des études conclue à une diminution des forces concentriques et excentriques développées quelle que soit l'articulation testée avec une plus large remise en cause des forces concentriques versus excentriques.

\subsubsection{Isocinétisme, adaptation et évolution}

Depuis de nombreuses années, des études concluent à une influence significative de l'avance en âge sur les forces concentriques et excentriques développées quelle que soit l'articulation testée (Porter, et al., 1995; Vandervoort, 1992).

Des travaux plus récents mettent en évidence la sensibilité des méthodologies d'évaluation isocinétique à caractériser les adaptations individuelles. Hambergvan Reenen, Visser, van der Beek, Blatter, van Dieën, \& van Mechelen (2009) étudient les désordres musculosquelettiques, le stress et la santé au travail à partir d'un suivi longitudinal de 1800 personnes. Les évaluations fonctionnelles sont réalisées sur les forces isocinétiques, l'endurance isométrique et la fonction aérobie et appliquées après trois ans de suivi. La sédentarité et la pratique d'une activité physique et sportive (APS) sont évaluées par questionnaire. Ils mettent en évidence des différences de capacités musculaires relatives à l'âge. Les jeunes travailleurs «actifs-fréquents » ont de meilleures capacités musculaires. Pour les travailleurs plus âgés, la participation à des APS modérées semble efficace face aux volumes de travail demandé.

Dans le domaine du vieillissement, les problématiques de sédentarité et de reconditionnement par des programmes d'activités physiques sont régulièrement traitées. Nous proposons maintenant, la présentation de résultats préliminaires d'un suivi de femmes sédentaires ostéopéniques réentraînées durant six mois et réévaluées à 12 mois post-entraînement.

\subsection{Méthode}

\subsubsection{Population}

Notre population était composée de 20 femmes sédentaires et ostéopéniques au début de l'étude. Les valeurs moyennes et d'écart type pour l'âge, le poids et la taille étaient respectivement de 67,4 ans $( \pm 4,2), 61,5 \mathrm{~kg}$ $( \pm 7,6)$ et $162,2 \mathrm{~cm}( \pm 6)$. Les critères d'inclusion dans ce programme Acti'march ${ }^{\circledR}$ adapté sur six mois étaient relatifs au genre féminin, à un âge situé entre 60 et 75 ans, à l'absence d'ostéoporose et de traitement interférant avec 


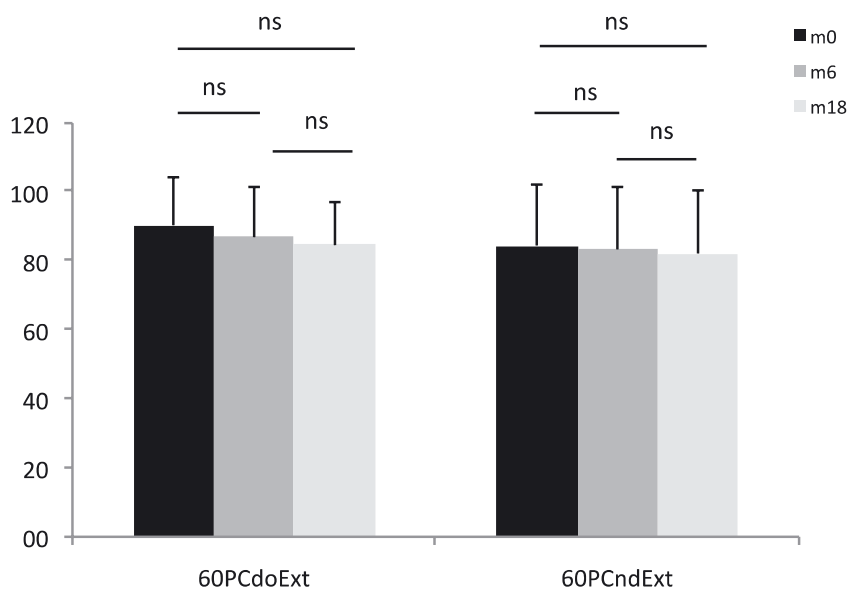

Fig. 2. Valeurs moyennes de pic de couple (PC) des extenseurs de genou mesurées à $60^{\circ} / \mathrm{s}$ du côté dominant (doExt) et non-dominant (ndExt) aux trois temps de début d'entraînementraînement $(\mathrm{m} 0)$, à 6 mois $(\mathrm{m} 6)$ et à 12 mois postentraînement (m18).

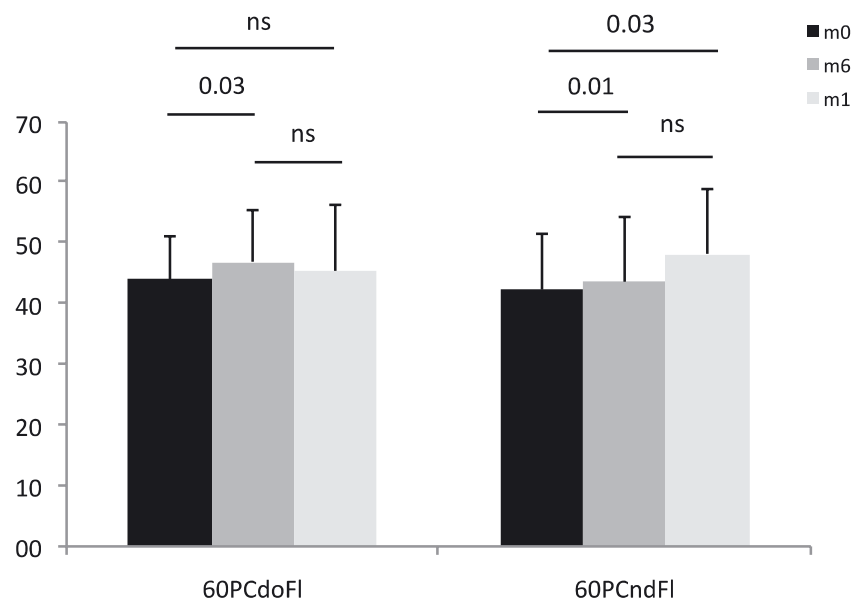

Fig. 3. Valeurs moyennes de pic de couple (PC) des fléchisseurs de genou mesurées à $60^{\circ} / \mathrm{s}$ du côté dominant (doFl) et non-dominant (ndFl) aux trois temps de début d'entraînement $(\mathrm{m} 0)$, à 6 mois $(\mathrm{m} 6)$ et à 12 mois post-entraînement (m18).

la mise en évidence d'évolutions fonctionnelles. La noninclusion était justifiée par la présence de pathologies lomocotrices et de troubles neuro-cognitifs.

\subsubsection{Protocole d'évaluation isocinétique}

Les évaluations isocinétiques étaient réalisées à l'inclusion avant le début du réentraînement $(\mathrm{m} 0)$, à la fin du réentraînement soit six mois plus tard (m6) et 12 mois après la fin du réentraînement soit 18 mois après l'inclusion $(\mathrm{m} 18)$ (Figs. 2 et 3 ).

Les femmes étaitent évaluées sur dynamomètre isocinétique Biodex Système $3{ }^{\circledR}$ avec correction de la gravité. L'évaluation était réalisée en mode concentrique/ concentrique sur une amplitude articulaire de $80^{\circ}$ avec placement de l'appui jambier à deux doigts sus-maléolaire et des butées placées respectivement à $-10^{\circ}$ en retrait de la verticale et $70^{\circ}$ d'extension. Un sanglage du tronc et de la cuisse active, un angle de $110^{\circ}$ entre le tronc et les cuisses et les membres supérieurs croisés sur le tronc étaient imposés. Chaque personne était soumise à un échauffement standardisé de deux séries de dix répétitions à $180^{\circ} / \mathrm{s}$ séparées d'une minute de récupération. Une période de trois minutes espaçait la fin de l'échauffement de l'application du premier test. Le protocole d'évaluation débutait par une série de cinq répétitions à $60^{\circ}$ / s puis 60 secondes de récupération avant cinq nouvelles répétitions à $180^{\circ} / \mathrm{s}$. Cinq minutes étaient nécessaires à l'installation de la personne pour l'évaluation du côté opposé, soumis aux mêmes conditions de test. Le paramètre de pic de couple (N.m) a été retenu pour l'analyse des résultats.

\subsubsection{Protocole d'entraînement}

Le protocole de réentraînement est issu du programme Acti'March ${ }^{\circledR}$. D'une durée de six mois à raison de deux séances collectives hebdomadaires de marche active et d'une séance individuelle en autonomie, il s'appuie sur des intensités d'effort personalisées de 45 à $80 \%$ de la fréquence cardiaque de réserve. Durant chaque séance d'une durée de l'ordre de 45 minutes, les variables de fréquence cardiaque, de durée d'effort et de distance pemettent une gestion de l'exercice.

\subsubsection{Analyse statistique}

Après vérification de la normalité de la distribution, les valeurs moyennes et de déviation standard ont été calculées pour l'ensemble des variables. Le test non-paramétrique de Wilcoxon pour séries appariées a été retenu pour la comparaison des distributions deux à deux. Un niveau de significativité de $p<$ 0,05 a systématiquement été retenu pour l'ensemble des analyses.

\subsection{Résultats préliminaires et analyse}

Pour les quadriceps (Fig. 2) à $60^{\circ} / \mathrm{s}$ du côté dominant, l'observation des valeurs de pic de couple met en évidence une absence de différence significative lors de la comparaison une à une des trois périodes de suivi. Lors de la comparaison des valeurs mesurées au début du programme et à six mois nous n'observons pas d'évolution significative et même une légère décroissance de la valeur moyenne de force maximale des quadriceps. À 12 mois post-entraînement, nous n'observons pas de décroissance significative par rapport aux valeurs mesurées à la fin de l'entraînement et une absence de différence significative par rapport au début du protocole situé à 18 mois. 
Du côté non-dominant nous observons des évolutions similaires c'est-à-dire une absence de différence significative aux trois temps avec une tendance à la décroissance au cours du temps. S'il est satisfaisant d'observer qu'à 12 mois post-entraînement, les valeurs de force musculaire n'ont pas significativement diminué il est plus décevant d'observer que six mois d'entraînement de marche active à raison de deux à trois entraînements hebdomadaires, n'ont pas amélioré la force développée.

Pour ce qui concerne les ischio-jambiers (Fig. 3), du côté dominant, l'observation des valeurs mesurées au début du programme et à six mois montre une augmentation significative $(p<0,03)$ de force maximale développée. À 12 mois post-entraînement, nous n'observons pas de différence notable par rapport aux valeurs mesurées à la fin de l'entraînement (six mois) et une absence de différence manifeste par rapport aux valeurs de début de protocole (18 mois). Du côté non dominant, nous observons une augmentation flagrante des valeurs de force mesurées à six mois $(p<0,01)$, une absence de différence significative entre six et 12 mois post-entraînement mais une augmentation notable entre le début de l'étude et 12 mois post-entraînement $p<0,03$ ).

Les adaptations des deux groupes musculaires extenseurs et fléchisseurs du genou s'avèrent donc différentes. Il s'agit de résultats préliminaires d'une étude plus vaste mais ils sont le support d'un certain nombre de questionnements à la fois méthodologiques pour ce qui est des évaluations isocinétiques et pratiques dans le cadre de l'entraînement.

- La vitesse de $60^{\circ} / \mathrm{s}$ est-elle la plus indiquée? Travailler à vitesse lente est nécessaire pour caractériser les plus hauts niveaux de force maximale mais peut mettre la personne en difficulté face à une intense résistance au mouvement. La tester à vitesse rapide est moins contraignant mais les qualités de vélocité sont elles aussi altérées par l'âge. Aussi, pour des personnes âgées faut-il privilégier une vitesse intermédiaire de l'ordre de 120 à $150^{\circ} / \mathrm{s}$ ?

- Rester centré sur les forces et les puissances lors de séries courtes, de l'ordre de cinq répétitions n'est-il pas une erreur ou au moins une limite? Certes les paramètres de $\mathrm{PC}$ et de $\mathrm{PM}$ sont parmi les plus valides mais l'évaluation de la capacité globale de travail témoignée par le paramètre de travail total est certainement pertinente. Dans de tels programmes de réentraînement pour des personnes âgées, l'analyse de travail total et le calcul de l'indice de fatigue par le rapport des dix dernières répétions sur les dix premiers au sein d'une série de 30 mouvements, constitueraient un apport significatif. Cela nécessite une session de test supplémentaire mais enrichit la compréhension des adaptations suite au reconditionnement.

- Ces résultats préliminaires permettent aussi d'interroger la programmation et les contenus d'entraînement. L'activité était centrée sur la marche et la gestion de l'effort sur la fréquence cardiaque avec des intensités cibles à atteindre. Par l'analyse à six mois (publications en cours), nous avons mis en évidence un bénéfice sur la fonction aérobie. En revanche, par l'évaluation isocinétique et les résultats observés à $60^{\circ} / \mathrm{s}$ des forces et des puissances, nous pouvons nous interroger sur les contenus qui seraient favorables à une optimisation plus significative de ces dimensions musculaires.

Remerciements. Pour la troisième étude, les auteurs souhaitent remercier la Fédération française d'éducation physique et de gymnastique volontaire (FFEPGV) pour le partenariat établi, le Comité régional EPGV du LanguedocRousillon en la personne de mesdames P. André et A.M. Garbal et enfin madame A. Bussone pour le remarquable travail d'animation réalisé au sein des ateliers.

\section{Bibliographie}

Allen, G.M., Gandevia, S.C., \& Mc Kenzie, D.K. (1995). Reliability of measurements of muscle strength and voluntary activation using twitch interpolation. Muscle and Nerve, 18, 593-600.

Baechle, T.R. (2008). Essentials of strength and conditioning. Third Edition. Champaign, Illinois: Human Kinetics, pp. 393-427.

Behm, D.G., Anderson, K., \& Curnew, R.S. (2002). Muscular force and activation under stable and unstable conditions. Journal of Strength and Conditioning Research, 16, 41622 .

Bernard P.L., Seynnes, O., \& Blain, H. (2010). Fatigue musculaire et vieillissement. In Dupeyron A., Perrey S., Herisson C. (Eds.), Fatigue musculaire. Paris : Masson.

Bernard P.L., Amato, M., Degache, F., Edouard, P., Ramdani, S., Blain, H., Calmels, P., \& Codine, P. (2012). Reliability of the time to peak torque and the joint angle at peak torque of the isokinetic dynamometer. Annals of Physical and Rehabilitation Medecine, 55, 241-251.

Bernard P.L., Edouard P., Tallon G., Perrey S., Billot M., Blain H, Codine P., Degache F. (2012). Influence of recovery time on strength during an isokinetic testing protocol of knee in middle-aged subjects. Isokinetics and Exercise Science (article submitted).

Bohannon, R.W., \& Smith, M.B. (1989). Intrasession reliability of angle specific knee extension torque measurements with gravity corrections. Journal of Orthopedic and Sports Physical Therapy, 11, 155-157.

Bottaro, M., Russo, A., \& Oliveira, R.J. (2005). The effects of rest interval on quadriceps torque during an isokinetic testing protocol in elderly. Journal of Sports Sciences and Medecine, 4, 285-290

Capranica, L.M., Battenti, M., Demarie, S., \& Figura, F. (1998). Reliability of isokinetic knee extension and flexion testing in elderly women. Journal of Sports Medecine and Physical Fitness, 38, 169-176.

Casartelli, N., Müller, R., \& Maffiuletti, N.A. (2010). Validity and reliability of the Myotest accelerometric system for 
the assessment of vertical jump height. Journal of Strength and Conditioning Research, 24, 3186-3193.

Celes, R., Brown, L.E., Pereira, M.C.C., Junior, V.A.R., \& Bottaro, M. (2010). Gender muscle recovery during isokinetic exercise. International Journal of Sports Medecine, 31, 866-869.

Chaouchi, A., Haddad, M., Castagna, C., Wong, D.P., Kaouech, F., Chamari, K., \& Behm, D.G. (2011). Potentiation and recovery following low and high speed isokinetic contractions in boys. Pediatric Exercice Science 23, 136-150.

Croisier, J.L., \& Crielaard, J.M. (1999). Exploration isocinétique : analyse des paramètres chiffrés. Annales Réadaptation et de Médecine Physique, 42, 538-545.

Dipla, K., Tsirini, T., Zafeiridis, A., Manou, V., Dalamitros, A., Kellis, E., \& Kellis, S. (2009). Fatigue resistance during high-intensity intermittent exercise from childhood to adulthood in males and females. European Journal of Applied Physiology, 106, 645-653.

Doherty, T.J., Vandervoort, A.A., Taylor, A.W., \& Brown, W.F. (1993). Effects of motor unit losses on strenght in older men and women. Journal of Applied Physiology, 74, 868-874.

Doherty, T.J., Vandervoort A.A., \& Brown W.F. (1993). Effects of ageing on the motor unit: a brief unit. Canadian Journal of Applied Physiology, 18, 331-358.

Erim, Z., Faisal Beg, M., Burke, D., \& De Luca, C. (1999). Effects of aging on motor-unit control properties. Journal of Neurophysiology, 82, 2081-209.

Fleisig, G.S., Andrews, J.R., Dillman, C.J., \& Escamilla, R.F. (1995). Kinetics of baseball pitching with implications about injury mechanisms. American Journal of Sports Medecine, 23, 233-239.

Forbes, S.C., Raymer, G.H., Kowalchuk, J.M., Thompson, R.T., \& Marsh, G.D. (2008). Effects of recovery time on phosphocretaine kinetics during repeated bouts of heavy-intensity exercise. European Journal of Applied Physiology, 103, 665-675.

Frontera, W.R., Hugues, V.A., Dallal, G.E., \& Evans, W.J. (1993). Reliability of isokinetic muscle strength in 45- to 78-year-old men and women. Archive of Physical Medecine and Rehabilitation, 74, 1181-1185.

Frontera, W.R., Hughes, V.A., Fielding, R.A., Fiatarone, M.A., Evans, W.J., \& Roubenoff, R. (2000). Aging of skeletal muscle: a 12-yr longitudinal study. Journal of Applied Physiology, 88, 1321-1326.

Hamada, T., Sale, D.G., Mc Dougall, J.D., \& Tarnopolsky, M.A. (2003). Interaction of fibre type, potentiation and human knee extensor muscles. Acta Physiologica Scandinavia, 178, 165-173.

Hamberg-van Reenen, H.H., Visser, B., van der Beek, A.J., Blatter, B.M., van Dieën, J.H., \& van Mechelen, W. (2009). Age-related differences in muscular capacity among workers. International Archive of Occupational and Environnemental Health, 82 (9), 1115-1121.
Hislop, H.J., \& Perrine, J.J. (1967). The isokinetic concept of exercise. Physical Therapy, 47, 114-7.

Imamura, K., Ashida, H., Ishikawa, T., \& Fujii, M. (1983). Human major psoas muscle and sacrospinalis muscle in relation to age: a study by computed tomography. Journal of Gerontology, 38, 678-681.

Impellizzeri, F.M., Bizzini, M., Rampinini, E., Cereda, F., \& Maffiuletti, N.A. (2008). Reliability of isokinetic strength imbalance ratios measured using the Cybex Norm dynamometer. Clinical Physiology and Functional Imaging, 28, 113-119.

Julia, M., Dupeyron, A., Laffont, I., Parisaux, J.M., Lemoine, F., Bousquet, P.J., \& Herisson, C. (2010). Reproducibility of isokinetic peak torque assessments of the hip flexor and extensor muscles. Annals of Physical and Rehabilitation Medecine, 53, 293-305.

Kannus, P. (1994). Isokinetic evaluation of muscular performance: implications for muscle testing and rehabilitation. International Journal of Sports Medecine, 15, 11-18.

Kawamura, Y., Okazaki, H., O'Brien, P.C., \& Dych, P.J. (1977). Lumbar motoneurons of man: number and diameter histogram of alpha and gamma axons of ventral root. Journal of Neuropathology and Experimental Neurology, 36 (5), 853-860.

Laidlaw, D.H., Bilodeau, M., \& Enoka, R.M. (2000). Steadiness is reduced and motor unit discharge is more variable in old adults. Muscle and Nerve, 23, 600-612.

Lexell, J., \& Downham, D.Y. (1991). The occurrence of fiber type grouping in healthy human muscle: a quantitative study of cross-sections of whole vastus lateralis from men between 15 and 83 years. Acta of Neuropathology, 81, 377381.

Lexell, J. (1995). Human aging, muscle mass, and fiber type composition. Journal of Gerontology, 50, 11-16.

Lindinger, M.I., Mc Kelvie, R.S., \& Heigenhauser, G.J. (1995). $\mathrm{K}+$ and Lac-distribution in humans during and after high-intensity exercise: role in muscle fatigue attenuation? Journal of Applied Physiology, 78, 765-777.

Macaluso, A., Nimmo, M.A., Foster, J.E., Cockburn, M., Mc Millan, N.C., \& De Vito, G. (2002). Contractile muscle volume and agonist-antagonist coactivation account for differences in torque between young and older women. Muscle and Nerve, 25, 858-863.

Machado, M., Koch, A.J., Willardson, J.M., Pereira, L.S., Cardoso, M.I., Motta, M.K., Pereira, R., \& Monteiro A.N. (2011). Effect of varying rest intervals between sets of assistance exercises on creatine kinase and lactate dehydrogenase responses. Journal of Strength and Conditioning Research, 25 (5), 1339-1345.

Mc Ardle, W.D., Katch, F.I., \& Katch, V.L. (2006). Energy transfer. In Mc Ardle W.D., Katch F.I., Katch V.L. (Eds.), Exercice physiology: energy, nutrition and human performance (pp. 166-288). 3rd ed. Lippincott Williams \& Wilkins. 
Mc Comas, A.J. (1996). Squeletal muscle. Champaign, Illinois: Human Kinetics.

Madsen, O.R. (1996). Torque, total work, power, torque acceleration energy and acceleration time assessed on a dynamometer: reliability of knee and elbow extensor and flexor strength measurement. European Journal of Applied Physiology, 74, 206-210.

Negrete, R.J., Hanney, W.J., Kolber, M.J., Davies, G.J., Ansley, M.K., Mc Bride, A.B., \& Overstreet, A.L. (2010). Reliability, minimal detectable change, and normative values for tests of upper extremity function and power. Journal of Strength and Conditioning Research, 24, 33183325 .

Östenberg, A., \& Roos, H. (2000). Injury risk factors in female European football. A prospective study of 123 players during one season. Scandinavian Journal of Medecine Science Sports, 10, 279-285.

Parcell, A.C., Sawyer, R.D., Tricoli, V.A., \& Chinevere, T.D. (2002). Minimum rest period for strength recovery during a common isokinetic testing protocol. Medecine and Science in Sports and Exercice, 34, 1018-1022.

Paterson, D.H., Jones, G.R., \& Rice, C.L. (2007). Le vieillissement et l'activité physique : données sur lesquelles fonder des recommandations relatives à l'exercice à l'intention des adultes âgés. Applied Physiology and Nutrition Metabolism, 32, 75-121.

Pincivero, D.M., Lephart, S.M., \& Karunakara, R.G. (1997). Effects of rest interval on isokinetic strength and functional performance after short term high intensity training. British Journal of Sports Medecine, 31, 229-234.

Pincivero, D.M., Lephart, S.M., \& Karunakara, R.G. (1998). Effects of intrasession rest interval on strength recovery and reliability during intensity exercise. Journal of Strength and Conditioning Research, 12, 152-156.

Pincivero, D.M., \& Campy, R.M. (2004). The effect of rest interval length and training on quadriceps femoris muscle. Part I: knee extensor torque and muscle fatigue. Journal of Sports Medecine and Physical Fitness, 44, 111-118.

Porter, M.M., Myint, A., Kramer, J.F., \& Vandervoort, A.A. (1995). Concentric and eccentric knee extension strength in older and younger men and women. Canadian Journal of Applied Physiology, 20 (4), 429-439.

Pousson, M., Lepers, R., \& van Hoecke, J. (2001). Changes in isokinetic torque and muscular activity of elbow flexors muscles with age. Experimental Gerontology, 36, 16871698.

Rassier, D.E., \& Mc Intosh, B.R. (2000). Coexistence of potentiation and fatigue in skeletal muscle. Brazilian Journal of Medecine Biological Research, 33, 499-508.

Ratel, S., Duché, P., \& Williams, C.A. (2006). Muscle fatigue during high-intensity exercise in children. Sports and Medecine, 36, 1031-65.

Rice, C.L., Cunningham, D.A., Paterson, D.H., \& Lefcoe, M.S. (1989). Arm and leg composition determined by computed tomography in young and elderly men. Clinical Physiology, 9, 207-220.
Robinson, J.M., Stone, M.H., Johnson, R.L., Penland, C.M., Warren, B.J., \& Lewis, R.D. (1995). Effects of different weight training exercise/rest intervals on strength, power and high intensity exercise endurance. Journal of Strength Conditioning Research, 9, 216-221.

Rooney, K.J., Herbert, R.D., \& Balnave, R.J. (1994). Fatigue contributes to the strength training stimulus. Medecine Science Sports Exercice, 26, 1160-1164.

Saenz, A., Avellanet, M., Hijos, E., Chaler, J., Garreta, R., Pujol, E., Sandoval, B., Buen, C., \& Farreny, A. (2010). Knee isokinetic test-retest: a multicentre knee isokinetic test-retest study of fatigue protocole. European Journal of Physical Rehabilitation Medecine, 46, 81-88.

Schott, J., Mc Cully, K., \& Rutherford, O.M. (1995). The role of metabolites in strength training. II. Short versus long isometric contractions. European Journal of Applied Physiology, 71, 337-341.

Shrout, P.A.F., \& Fleiss, J.L. (1979). Intraclass correlation: uses in assessing rater reliability. Psychology Bulletin, 85, $420-428$.

Söderman, K., Alfredson, H., Pietilä, T., \& Werner, S. (2001). Risk factor for leg injuries in female soccer players: a prospective investigation during one out-door season. Knee Surgery Sports Traumatology Arthroscopy, 9, 313-321.

Stalberg, E., Borges, O., \& Ericsson, M. (1989). The quadriceps femoris muscle in 20-70-year old subjects: relations hip between kneee extension torque, electrophysiological parameters, and muscle fiber characteristics. Muscle and Nerve, 12 (5), 382-389.

Theou, O., Gareth, J.R., \& Brown, L.E. (2008). Effect of rest interval on strength recovery in young and old women. Journal of Strength and Conditioning Research, 22, 18761881.

Touey, P.R., Sforzo, G.A., \& Mc Manys, B.G. (1994). Effect of manipulating rest periods on isokinetic muscle performance. Medecine and Science in Sports and Exercice, 27, S170.

Vandervoort, A.A. (1992). Effects of aging of human neuromuscular function: implications for exercice. Canadian Journal of Sport Science, 17 (3), 178-184.

Westerblad, H., Allen, D.G., Bruton, J.D., Andrade, F.H., \& Lännergren, J. (1998). Mechanisms underlying the reduction of isometric force in skeletal muscle fatigue. Acta Physiologica Scandinavia, 162, 253-260.

Willardson, J.M. (2006). A brief review: factors affecting the length of the rest interval between resistance exercise sets. Journal of Strength and Conditioning Research, 20, 978984.

Wilmore, J.H., Costill, D.L., Kenney, W.L. (2008). Fuel for exercice. In: Wilmore J.H., Costill D.L., \& Kenney W.L. (Eds.), Physiology of Sport and Exercice. (pp. 49-68). Champaign, Illinois: Human kinetics, Fourth Edition. 